ORIGINAL ARTICLE

\title{
Do we know what people die of in the emergency department?
}

\section{F Mushtaq, D Ritchie}

See end of article for authors' affiliations

.....................

Correspondence to: Miss F Mushtaq, Specialist Registrar in Accident and Emergency Medicine, Crosshouse Hospital, Kilmarnock, KA2 OBE; farhatmushtaq@doctors. net.uk

Accepted for publication 26 October 2004

\begin{abstract}
Objective: To establish the discrepancy rate between the predicted cause of death and the actual cause of death as determined by postmortem examination result, for all deaths in the emergency department reported to the Scottish Procurator Fiscal and subsequently undergoing postmortem examination.

Methods: A prospective study of all patients who were dead on arrival or died in the emergency department of a busy Glasgow hospital over a 12 month period. The most senior emergency physician present at the time of death predicted the cause of death. This was then compared to the actual postmortem examination determined cause of death and was considered either to be correct or incorrect.

Results: During the study period, 146 patients were pronounced dead in the department. Of these, 81 patients (age range 39-99 years, median 71; male:female 2.5:1) had death certificates issued, 63 patients (age range 26 days to 99 years, median 48; male:female 2.4:1) had a postmortem performed by the forensic pathologist, and two patients underwent a "view and grant". Of the 63 deaths reported to the Procurator Fiscal, the emergency physician attributed 51 (80.1\%) to non-trauma, $9(14.2 \%)$ to trauma, and in $3(4.7 \%)$ cases were uncertain. Of the $63(39.7 \%)$ deaths, 25 were inaccurately predicted $199 \%$ confidence interval $24.3 \%$ to $56.6 \% ; \mathrm{p}<0.0) 1$. Cardiovascular related and drugs poisoning deaths occurred most commonly. They were also the most accurately predicted cause of deaths. Intracranial events, pulmonary thromboembolism, and airway obstruction were also frequently predicted, but were often wrong.

Conclusions: This study highlights the difficulties in accurately identifying cause of death for patients who die suddenly. This could have implications for the accuracy of health service statistics.
\end{abstract}

E mergency Departments (ED) continue to remain at the interface of community and hospital medicine. Many patients who die in the ED are often critically ill on arrival and their illness is often unexpected. It may be their first presentation to the department and consequently the patient may be unknown to the attending medical team. ${ }^{1}$

Available resources are used to gain further information regarding the cause of death. These include the past medical and presenting history of the patient from relatives and information from the ambulance crews, hospital notes, and the patient's general practitioner (GP). This information is taken in conjunction with the clinical presentation and allows the attending emergency physician to ascertain if a death certificate can be issued or if the death requires by law to be reported to the authorities, which in Scotland is the office of the Procurator Fiscal (PF). As the role of the PF in Scotland is primarily in the detection of criminality and negligence, the Crown Office in Scotland has issued a comprehensive set of guidelines to all medical departments indicating which deaths must be reported to the PF. ${ }^{2}$ These include any death due to violence, trauma, poisoning or suicide, and if the doctor is unable to certify the cause of death with reasonable certainty.

The aim of this study was to compare the predicted cause of death with the postmortem examination (PME) findings in patients who were pronounced dead in the ED, with the aim of highlighting the difficulty in accurately predicting cause of death.

\section{METHODS}

A 1 year prospective study was undertaken at the ED of Victoria Infirmary, Glasgow, in the period August 2002 to July 2003. The department has an annual attendance rate of approximately 70000 and serves a wide catchment area varying from affluent suburban areas to areas associated with marked social deprivation. For all patients who were declared dead in the department, data were collected concerning the clinical impressions of the most senior ED physician present at the time of the resuscitation as to the most likely cause of death. The ED physician's predicted cause of death was ascertained after further information was gained regarding the circumstances surrounding the patient's death and relevant background history.

After discussion with the patient's GP and/or the PF, the patients were subclassified into the following categories: (a) death certificate issued; $(b)$ "view and grant" by the PF; or $(c)$ PME requested by the PF.

All PMEs and "view and grants" were performed by forensic pathologists based at Glasgow University Department of Forensic Pathology, in accordance with Crown Office procedures. Information regarding the actual cause of death as recorded by the pathologist was later acquired from the Procurator Fiscal's Office.

F Mushtaq (the first author) was responsible for recording both the predicted cause of death and the PME cause of death; the latter was taken to be the "primary cause of death" as issued to the PF by the forensic pathologist. We both noted the discrepancies between the predicted cause of death by the attending clinician and the actual PME confirmed cause of death, and we had to agree in conjunction on the clinician's diagnosis as "correct" or "incorrect". This group of patients undergoing PME examination formed the main body of our study.

Abbreviations: $E D$, emergency department; GP, general pracitioner; $\mathrm{PF}$, Procurator Fiscal; PME, postmortem examination; PTE, pulmonary thromboembolism 


\begin{tabular}{|ll|}
\hline $\begin{array}{l}\text { Table } 1 \text { Primary cause of death on death } \\
\text { certificate }\end{array}$ & $\begin{array}{l}\text { No. of } \\
\text { patients }\end{array}$ \\
\hline \multicolumn{2}{|l|}{ Primary cause of death on death certificate } \\
\hline Cardiovascular system \\
Acute myocardial infarction & 40 \\
Cardiac arrhythmia & 10 \\
Left ventricular failure & 8 \\
Ischaemic heart disease & 3 \\
Respiratory system & 3 \\
Respiratory failure & 1 \\
Chronic obstructive airways disease & 1 \\
Asthma & 1 \\
Airway obstruction & 2 \\
Gastrointestinal system & 1 \\
Ruptured abdominal aortic aneurysm & 5 \\
Gastrointestinal haemorrhage & 3 \\
Carcinoma & 2 \\
Intracranial haemorrhage & 1 \\
Septicaemia & 81 \\
Old age & \\
Total & \\
\hline &
\end{tabular}

\section{Statistical analysis}

Results were analysed using SPSS for Windows (version 11; SPSS Inc., Chicago, IL, USA) and $\mathrm{p}<0.01$ was considered significant.

\section{RESULTS}

During the study period, 146 patients were pronounced dead in the ED (age range 26 days to 99 years, median 64; male:female 1.8:1). Only 5 of these 146 patients (3.4\%) had evidence of a palpable pulse on arrival. Death certificates were issued by the emergency physicians for 81 patients (age range 39-99 years, median 71; male:female 2.5:1). Table 1 illustrates the primary cause of death on the death certificates as issued by the emergency physicians. In total, 63 patients (age range 26 days to 99 years, median 48; male:female 2.4:1) had a PME performed by the forensic pathologist and 2 patients underwent a "view and grant".

Fig 1 illustrates the relative age group of deaths who underwent PME examinations. Of the 63 deaths reported to the $\mathrm{PF}$, the emergency physician attributed $51(80.1 \%)$ to non-trauma and $9(14.2 \%)$ to trauma. In three $(4.7 \%)$ deaths it was unclear whether the death was caused by trauma or non-trauma.

Table 2 demonstrates the non-trauma causes of death. These were classified into either major body systems or the pathological process leading to death. It demonstrates the frequency with which error occurred in correctly identifying the cause of death when compared with the PME report. Cardiovascular system and drug poisoning were most commonly predicted as a cause of death, and were correct in $80 \%$ of cases. In contrast, intracranial haemorrhages and airway obstruction were also frequently predicted, but were commonly wrong.

Table 3 indicates the PME ascertained causes of death for all deaths that had been incorrectly predicted. Cardiac related deaths were the most frequently predicted. On the 3 of 15 occasions when the prediction was incorrect, pulmonary thromboembolism (PTE) was the causative factor of death. Conversely, of the five patients who had PTE as the predicted cause of death, the three incorrectly diagnosed had in fact died from a cardiac related death. The remaining two deaths were correctly attributed to a PTE.

Drug poisoning was accountable for eight deaths in our study. Alcohol intoxication and fatty degeneration of the liver were the causes of death on the two occasions when drug poisoning was misdiagnosed.
There were six patients with an intracranial haemorrhage as a predicted cause of death. Only on one occasion was the emergency physician correct. Of the remaining five patients, two had died from an acute myocardial infarction, and one

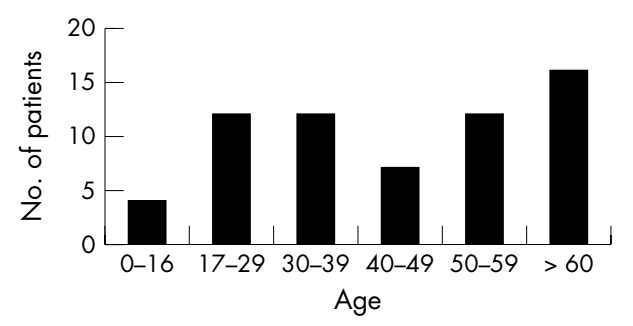

Figure 1 Age groups requiring PME.

Table 2 Anticipated cause of death compared with results of the PME

\begin{tabular}{lccc}
\hline Anticipated cause of death & Correct & Incorrect & \% accuracy \\
\hline Cardiovascular system & 12 & 3 & 80 \\
Pulmonary thromboembolism & 2 & 3 & 40 \\
Drugs poisoning & 8 & 2 & 80 \\
Intracranial haemorrhages & 1 & 5 & 17 \\
Seizure related deaths & 2 & 1 & 67 \\
Gastrointestinal system & 1 & 2 & 33 \\
Airway obstruction & 0 & 5 & 0 \\
Viral illness & 0 & 1 & 0 \\
Anaphylaxis & 0 & 1 & 0 \\
Carcinoma & 0 & 1 & 0 \\
Sudden adult death & 1 & 0 & 100 \\
Total & 27 & 24 & 53 \\
\hline
\end{tabular}

Table 3 Anticipated cause of death versus PME ascertained cause of death for the incorrect diagnoses

\begin{tabular}{|c|c|}
\hline $\begin{array}{l}\text { (Incorrect) anticipated } \\
\text { cause of death }\end{array}$ & PME ascertained cause of death \\
\hline \multicolumn{2}{|l|}{ CVS } \\
\hline Ischaemic heart disease & Pulmonary thromboembolism \\
\hline Ischaemic heart disease & Pulmonary thromboembolism \\
\hline Acute myocardial infarction & Pulmonary thromboembolism \\
\hline \multicolumn{2}{|l|}{ Drug poisoning } \\
\hline Heroin overdose & Alcohol intoxication \\
\hline Heroin overdose & Fatty degeneration of liver \\
\hline \multicolumn{2}{|l|}{ Pulmonary thromboembolism } \\
\hline Pulmonary thromboembolism & Acute myocardial infarction \\
\hline Pulmonary thromboembolism & Ischaemic heart disease \\
\hline Pulmonary thromboembolism & Ischaemic heart disease \\
\hline \multicolumn{2}{|l|}{ Intra-cranial haemorrhages } \\
\hline Subarachnoid haemorrhage & Intra-abdominal haemorrhage \\
\hline Subarachnoid haemorrhage & Acute myocardial infarction \\
\hline Subarachnoid haemorrhage & $\mathrm{HOCM}$ \\
\hline Cerebrovascular accident & Pulmonary thromboembolism \\
\hline Cerebrovascular accident & Ischaemic heart disease \\
\hline Seizure related & Bronchopneumonia \\
\hline \multicolumn{2}{|l|}{ Gastrointestinal system } \\
\hline GI haemorrhage & Cardiac enlargement \\
\hline Bowel ischaemia & Ischaemic heart disease \\
\hline Viral illness & Dissected thoracic aneurysm \\
\hline \multicolumn{2}{|l|}{ Airway obstruction } \\
\hline Mechanical AO & Amphetamine induced heart failure \\
\hline Asphyxiation & Pneumonia \\
\hline Aspiration & $\begin{array}{l}\text { Sudden unexpected death in } \\
\text { epilepsy }\end{array}$ \\
\hline Aspiration & Ischaemic heart disease \\
\hline Aspiration & Acute myocardial infarction \\
\hline Anaphylactic reaction & Acute asthma attack \\
\hline Carcinoma & Ischaemic heart disease \\
\hline
\end{tabular}


each from intra-abdominal haemorrhage, PTE, and hypertrophic obstructive cardiomyopathy (HOCM).

On five occasions, patients had their deaths incorrectly attributed to airway obstruction. PME ascertained causes of death for this group included amphetamine induced heart failure, pneumonia, sudden unexpected death in epilepsy, ischaemic heart disease, and AMI.

Of the nine deaths due to trauma, the anticipated cause of death was correct on all nine occasions. On four occasions patients had been the victims of vehicular related trauma, three patients died from penetrating knife injuries, one had fallen from a height, and one had committed suicide by hanging.

For the three deaths where it was unclear whether the death was due to trauma/non-trauma, the cause of death was predicted correctly on two occasions. All three of these patients were elderly and had been found lying on the floor, with evidence of a head injury. Of these PMEs revealed AMI as the cause of death in two patients, and in the remaining patient death was attributed to a traumatic intracranial haemorrhage.

Overall, $25(39.7 \%)$ of the 63 patients (99\% confidence interval $24.3 \%$ to $56.6 \%$; $<<0.01$ ] had their cause of death inaccurately predicted by the ED physician.

\section{DISCUSSION}

Despite a widespread fall in the hospital PME rate in the UK, the number of referrals to the coroner in England and Wales is on the increase and is expected to continue to rise following the Shipman Inquiry, ${ }^{3-6}$ which was instigated following the conviction of a doctor in the UK for the murder of a large number of patients in his care.

Death rates in England and Scotland are approximately 1\% of the population per year. In Scotland, $24 \%$ of deaths are reported to the PF, while $35.5 \%$ are referred to the coroner in England and Wales. ${ }^{7}$ A PME examination is performed in $41 \%$ of deaths reported to the PF and $67.7 \%$ reported to the coroner. ${ }^{7}$

Pathologists in Scotland are able to perform a "view and grant", where knowledge of the history and circumstances of the death accompanied by a full external examination of the body allows a death certificate to be issued. This accounts for approximately $10 \%$ of the workload and is invariably confined to deaths due to natural causes. ${ }^{7}$

There is no doubt that PME remains the gold standard by which physicians' clinical diagnosis is either confirmed or contested. ${ }^{8}$ However, Rutty and his colleagues also demonstrated that even pathologists, by analysing available information before PME, also frequently misdiagnose the true cause of death. ${ }^{5}$

It has been recognised that cardiac related deaths, especially AMI, are often overpredicted, and that AMI can be a convenient and rarely contested label to use. ${ }^{4}$ Quigley et al highlighted that almost a third of deaths in their ED had death certificates issued as cardiac related death, when in fact there was no concrete evidence to support this. ${ }^{9}$ For death certificates issued by the emergency physicians in our ED, 40 of the 81 patients had an AMI as the primary cause of death. Although cardiac related deaths constituted a large proportion of death certificates issued, it is our ED policy that adequate information is sought and obtained by reviewing the circumstances leading to all deaths and using additional resources of information such as the attending emergency services personnel, family members, hospital notes, and previous history from the GP.

In our study of patients on whom a PME was performed, cardiac related deaths were overpredicted by $20 \%$. These patients at PME were found to have died from a PTE, thus highlighting the similarity of the suddenness in both events and the difficulty in clinically distinguishing between the two conditions.
Patients whose predicted cause of death was anticipated to be of non-cardiac or non-poisoning origin were often wrongly predicted. "Common" causes of death such as cardiac related deaths were frequently found at PME; however, uncommon causes of death that were not anticipated were also found, such as dissected thoracic aneurysm, amphetamine induced heart failure, and HOCM. The latter case suggests that sudden death often has implications for the family involved, both in terms of risk prediction and regarding possible "screening" of remaining family members.

Recent high profile media cases highlighting the scandal of retained organs has had a dramatic fall in the number of PMEs performed in the UK as a whole. As a result relatives are often reluctant to give permission for a PME, because they feel that the deceased has been through enough and it will be of no benefit to them. ${ }^{10}$ The ED in this situation is a unique setting, because by law we are required to report certain categories of death.

As emergency physicians, we need to use the remaining resources available to us, such as PF ordered PMEs to look at clinical diagnoses missed and whether our initial management plan should have been different for these patients. In addition, PMEs can also be used to confirm a diagnosis.

In our study, only 5 of the 146 patients had a pulse on arrival to the ED. These five patients had death certificates issued by the ED. We do not believe that the outcome for any patient would have been different had we known the initial diagnosis. Indeed, the study highlights that patients who arrive in the ED without a pulse have suffered some kind of irreversible catastrophic event, and despite appropriate advanced cardiac or trauma life support, the outcome remains dismal. As a result of the suddenness of the event, it is difficult for the emergency physician to accurately ascertain the cause of death.

Studies have indicated that whether the patient has been in hospital for minutes, hours, or weeks before death, the cause of death remains difficult to predict accurately and major discrepancies between the anticipated cause of death and the PME cause of death remain. ${ }^{8}{ }^{10}$ Our study and the studies quoted leads us to the conclusion that the only reliable way of measuring the prevalence, incidence, mortality, and morbidity of a disease is by PME rather than death certification by the attending physician.

PME rates approaching $100 \%$ will not be attainable. We must therefore be aware of the wider implications for health statistics, as the diagnosis on death certificates are used in the planning of health services and epidemiological research. ${ }^{11}$

We believe that in the meantime, for any patient who undergoes a PME following a sudden and inexplicable death, ED physicians should ascertain their cause of death. This information can be used to audit any potential areas for clinical improvement.

\section{Limtations}

One limitation of the study is that the group of patients that had a death certificate issued is likely to differ from the PME patient group in the information available and the inherent predicted diagnostic accuracy, therefore the latter group is highly selected. In addition, the grade of the emergency physician who predicted the cause of death varied from an experienced senior house officer in emergency medicine to a consultant in emergency medicine. However, our aim was not to highlight the differences in prediction rates between grades of emergency physician, but rather to emphasise the difficulty in accurately predicting a cause of death.

One possible source of bias could be the interobserver variability amongst the ED physicians. Although we did not address this directly in the study, having looked at the data we do not think this was a major factor. 


\section{ACKNOWLEDGEMENTS}

We would like to thank all the emergency physicians at the Emergency Department, Victoria Infirmary for taking part in the study.We would also like to thank the staff at the The Deaths Unit, Procurator Fiscal's Office for providing us with the cause of deaths, J Henry for the statistical analysis, and C McGuffie and T Mushtaq for their comments on the manuscript.

\section{Authors' affiliations}

F Mushtaq, Crosshouse Hospital, Kilmarnock, UK

D Ritchie, Victoria Infirmary, Langside Road, Glasgow, UK

Competing interests: none declared

\section{REFERENCES}

1 Whitehouse SR, Kisson N, Singh N, et al. The utility of autopsies in a paediatric emergency department. Paed Emer Care 1994;10:72-5.

2 The Scottish Office Department of Health. Death and the procurator fiscal The Scottish Office Department of Health, 1998.

3 Inglis FG, McMurdo MET. Post-mortem rates and junior doctors in Tayside Three years after the Joint Working Party Report. Health Bulletin, 1995:53:379-85.

4 Smith CJ, Scott SM, Wagner BM. The necessary role of autopsy in cardiovascular epidemiology. Human Pathology 1998;29:1469-78.

5 Rutty GN, Duerden RM, Carter N, et al. Are coroners' necropsies necessary? A prospective study examining whether a 'view and grant' system of death certification could be introduced into England and Wales. J Clin Pathol 2001;54:279-84.

6 The Shipman Inquiry. A discussion paper. Developing a new system for death certification, October, 2002

7 Davidson AM, McFarlane JH, Clark JC. Differences in forensic pathology practice between Scotland and England. Med Sci Law 1998;39:283-8.

8 Stothert JC, Gbaanadot GBM, Herndon DN. The role of autopsy resulting from trauma. J Trauma 1990;30:1021-6.

9 Quigley M, Burton J. Evidence for cause of death in patients dying in an accident and emergency department. Emerg Med J 2003;20:349-51.

10 Wright JC, Grant IS. Evaluation of the autopsy following intensive care. Intensive Care, 2001; Spring, 6-8.

11 Kircher T, Nelson J, Burdo H. The autopsy as a measure of accuracy of the death certificate. N Engl J Med 1985;313:1263-9. 